\title{
Novel Approach to Cluster Synchronization in Kuramoto Oscillators
}

\author{
Xin Biao Lu \\ Department of Automation, College of Energy and Electrical Engineering, Hohai University, Nanjing, China \\ Email: xinbiaolu@126.com \\ Bu Zhi Qin \\ Department of Automation, Nanjing College of Chemical Technology, Nanjing, China \\ Email: buzhiqin@126.com
}

\begin{abstract}
Cluster synchronization is investigated in different complex dynamical networks. Based on an extended Kuramoto model, a novel approach is proposed to make a complex dynamical network achieve cluster synchronization, where the critical coupling strength between connected may be obtained by global adaptive approach and local adaptive approach, respectively. The former approach only need know each node's state and its destination state; while the latter approach need know the local information. Simulation results show the effectiveness of the distributed control strategy.
\end{abstract}

Index Terms-cluster synchronization, global approach, local approach, Kuramoto model

\section{INTRODUCTION}

Kuramoto model and its extensions are widely used in the research of phase synchronization [1-7]. In the Kuramoto model, all phase oscillators are coupled, in addition all the edge strength are equal; however, its extensions have more general network topology and edge strength [1-2]. For more details, refer the review paper [1] and its references. Phase synchronization of two typical network topologies: small world network and scale-free network are investigated in [3-5]. However, in these works, the edge strength is symmetrical. By introducing nonsymmetrical coupled edge strength, $\mathrm{Li}$ found for a random network topology, the network has same critical coupling strength as the classical Kuramoto model [6]. During the formation of phase synchronization, people find that the oscillators always first form several synchronized clusters, then as the coupling strength increases, the whole oscillators reach synchronization [78]. This drives Belykh et al. to the existence and stability of these synchronized clusters [9]. In these networks, the coupling strength can not be tuned. However, for some purposes, the tunable coupling strength is necessary. For example, when a crowd of robots rescue several wounded persons in the fire, the robots must be divided into several groups in order to save time, the robots in the same group rescue one person, respectively. For the unexpected things happen such as one robot loses a sensor, the coupling strength between robots must be adjusted timely.
Recently, adaptive method is applied to investigate the synchronization of complex networks [10-15]. When the isolated node's dynamics is chaotic oscillator, Kurths et al. proposed time-varying adaptive coupling strength, which depends on global information in the network [10]. Furthermore, Lellis et al. and Lu supposed time-varying adaptive coupling strength based on the local information in the network, respectively [12-14]. When the isolated node's dynamics is Kuramoto oscillator, Ren and Zhao designated an adaptive law based on the local information in the network [15]. These adaptive laws are only applied to complete synchronization and phase synchronization. This propels us to design the adaptive strategy applied to cluster synchronization.

In this paper, a novel distributed control strategy is proposed to make the network achieve cluster synchronization. Two adaptive approaches are used to obtain the critical coupling strength between nodes. In the former approach, the edge strength between nodes is assumed to be equal, and adjusts according to each node's state and its destination; while in the latter approach, the edge strength is different, and adjust only with local information. When the underlying network is classical BA scale-free network, WS small-world network and a friendship network, the effectiveness of the distributed strategy and two adaptive approaches are verified by simulation results.

\section{AdAPTIVE APPOACH OF KURAMOTO MODEL}

Consider an extend Kuramoto model of $N$ coupled phased identical nodes, the evolution of the dynamical variable is written as follows:

$$
\begin{gathered}
\dot{\theta}_{i}(t)=\omega_{i}+\sum_{j=1, j \neq i}^{N} w_{i j} a_{i j}(t) \sin \left(\theta_{j}(t)-\theta_{i}(t)-u_{i}(t)\right), \\
i=1,2, \cdots, N,
\end{gathered}
$$

where $\omega_{i}$ is natural frequencies distributed with a given probability density. $u_{i}(t)$ is a control input added to each node. When the control input $u_{i}(t)$ equals zero, (1) reduces to the classical extend Kuramoto model 
investigated in [2]. $W=\left(w_{i j}\right)_{N \times N}$ is the matrix of the coupling strength between nodes. If there is a connection between node $i$ and node $j$, then $w_{i j}=w_{j i}>0(i \neq j)$, otherwise $w_{i j}=w_{j i}=0(i \neq j)$. $A=\left(a_{i j}\right)_{N \times N}$ is the connection matrix. If there is a connection between node $i$ and node $j$, then $a_{i j}=a_{j i}=1(i \neq j)$, otherwise $a_{i j}=a_{j i}=0(i \neq j)$.

Assume $N$ nodes finally achieve $M$ - cluster synchronization and the whole nodes splits into $M$ clusters. Without loss of generality, the sets of subscripts of these clusters are $G_{1}=\left\{1,2, \cdots, N_{1}\right\}$, $G_{2}=\left\{N_{1}+1, N_{1}+2 \cdots ; N_{1}+N_{2}\right\}, \cdots, G_{M}=\left\{N_{1}+\cdots+N_{M-1}+1, \cdots ; N\right\}$, where $N_{1}+N_{2}+\cdots+N_{M}=N$.

When $t \rightarrow \infty$, if the network reaches the cluster synchronization manifold $C S=\left\{x_{1}(t)=\cdots=x_{N_{1}}(t), x_{N_{1}+1}(t)=\cdots=x_{N_{1}+N_{2}}(t), \cdots, x_{N_{1}+\cdots+N_{M-1}+1}(t)=\cdots=x_{N}(t)\right.$ , the network is said to achieve cluster synchronization. The CS considered in this paper is defined as

$$
C S=\left\{\begin{array}{l}
s_{1}(t)=\cdots=s_{N_{1}}(t) ; s_{N_{1}+1}(t)=\cdots \\
=s_{N_{1}+N_{2}}(t) ; \cdots, s_{N_{1}+\cdots+N_{M-1}+1}(t) \\
=\cdots=s_{N}(t)
\end{array}\right\}
$$

where $s_{i}(t)=\left(s_{i 1}(t), \cdots, s_{i n}(t)\right)^{T}$ is the desired state of node $i$ at time $t$, and satisfies $\dot{s}_{i}(t)=f\left(s_{i}(t), t\right)$.

In order to make the network achieve cluster synchronization manifold (3), a novel distributed controller is designed as follows:

$$
u_{i}(t)=s_{i}(t)-s_{j}(t)
$$

Obviously, if node $i$ and node $j$ are in a same group, then $s_{i}(t)=s_{j}(t)$ and the contribution of the link between them to the input $u_{i}(t)$ is zero. Furthermore, when there is only one group in the network, the input $u_{i}(t)$ is zero.

\section{A Global adaptive approach}

In the global adaptive approach, the edge strength of all edges is equal, which means that $w_{i j}(t)=w$ for any $w_{i j}>0$. The edge strength is adjusted by the following adaptive strategy.

$$
\dot{w}(t)=\beta \sum_{i=1}^{N}\left|\theta_{i}(t)-s_{i}(t)\right|
$$

where $\beta>0$ is the adaptive gain. Obviously, the global adaptive approach does not need know the detailed connection between nodes.

$B$ Local adaptive approach

The $a_{i j}(t)$ is edge strength and is adjusted by the following adaptive strategy.

$$
\dot{w}_{i j}(t)=\beta \mid \sin \left(\left(\theta_{j}(t)-s_{j}(t)\right)-\left(\theta_{j}(t)-s_{j}(t)\right) \mid\right.
$$

Compared to the global adaptive approach, the local approach only need know the states of the relate nodes and their destinations. Obviously, if there is only one group in the network, then for random node $i$ and node $j, s_{i}=s_{j}$ Furthermore, equation (5) becomes

$$
w_{i j}(t)=\beta\left|\sin \left(\theta_{j}(t)-\theta_{j}(t)\right)\right|
$$

Then, (7) is the adaptive strategy used in [15].

\section{SIMULATIONS}

Two classical complex networks are constructed to verify the effectiveness of the proposed local adaptive approach and global adaptive approach, respectively.

\section{A Global adaptive approach in BA scale-free network}

At first, a BA scale-free network is constructed with $m=m_{0}=5$ and $N=100$ [16]. The whole nodes of the investigated network are randomly divided into three groups, which means $M=3$. The desired state $s_{i}(t)$ of node $i$ at time $t$ is chosen as the average of the nodes' states in the same group:

$\left\{\begin{array}{l}S_{1}(t)=S_{2}(t)=\cdots=S_{N_{1}}(t)=\frac{\theta_{1}(t)+\cdots+\theta_{N_{1}}(t)}{N_{1}} \\ S_{N_{1}+1}(t)=S_{N_{1}+2}(t)=\cdots=S_{N_{1}+N_{2}}(t)=\frac{\theta_{N_{1}+1}(t)+\cdots+\theta_{N_{1}+N_{2}}(t)}{N_{2}} \\ S_{N_{1}+N_{2}+1}(t)=S_{N_{1}+N_{2}+2}(t)=\cdots=S_{N}(t)=\frac{\theta_{N_{1}+N_{2}+1}(t)+\cdots+\theta_{N}(t)}{N_{3}}\end{array}\right.$,

where three group size $N_{1}, N_{2}$ and $N_{3}$ are 24, 38 and 38, respectively. The nodes' initial phases are randomly distributed in $[0,2 \pi]$ and the initial edge strengths are set to zero.

Figure 1 shows that cluster synchronization in Kuramoto oscillators, where the underlying network is BA scale-free network. The distributed controller (4) is added to each node, and the edge strength is adjusted according to (5) with the adaptive gain $\beta=0.1$. As can be seen from Fig. 1(a), for $t>2.4 s, 24$ dashed lines reduce to one dashed line, 38 dotted line reduces to one dotted line, and the rest 38 solid lines reduce to one solid line. This implies that the network reaches the desired cluster synchronization. 
It is easy to find that in Fig. 1(b) that the edge strength $w(t)$ keeps constant for $t>2.4 \mathrm{~s}$. Combined with Fig. 1(a), it is found that the network reaches cluster synchronization for $t>2.4 \mathrm{~s}$, which implies that the left side of (5) is zero. In other words, the edge strength will not increase any more in theory, which is consistent with the simulation result in Fig.1.

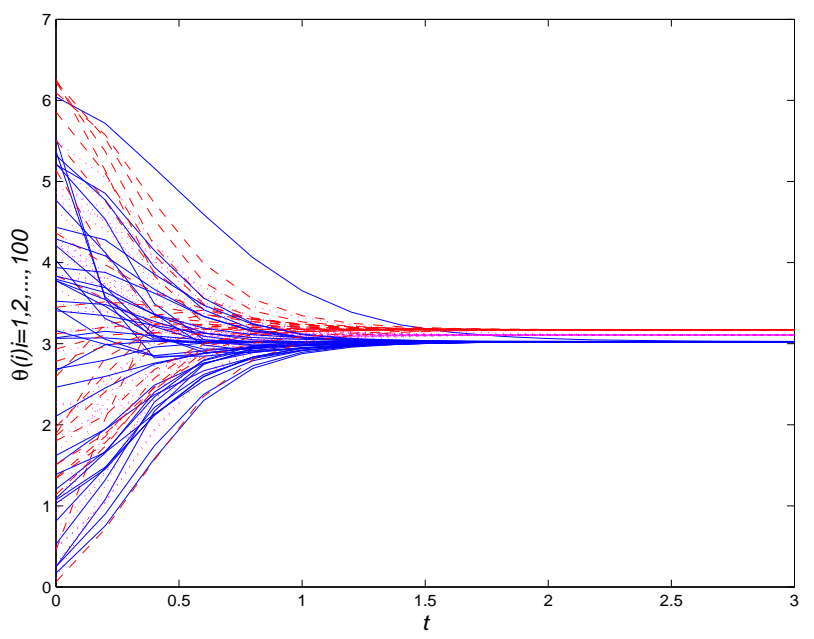

(a)

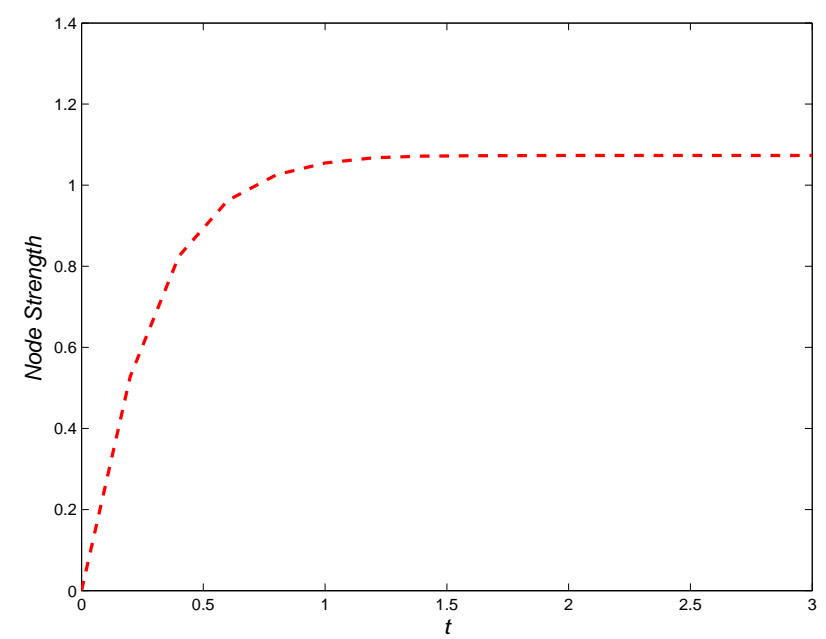

(b)

Fig. 1 Cluster synchronization in the Kuramoto oscillators, where the underlying network is BA scalefree network without noise and the global adaptive gain $\beta=0.1$. (a) $\theta_{i}(t)$ (b)edge strengths $w(t)$.

It is known that the noise is often encountered in many real world complex networks. Therefore, the effect of noise on the global adaptive approach is important. The network used in Fig.2 is the same as that used in Fig.1.
An independent noise $\alpha \psi_{i}$ is added to the variable of (1) respectively at $t=3 \mathrm{~s}$ in Fig. 2, where the amplitude of the noise $\alpha$ is $2 ; \psi_{i}$ randomly satisfies the normal Gaussian distribution $N(0,1)$.

As shown in Fig.2(a), the network reaches cluster synchronization again for $t>4.8 \mathrm{~s}$. At the same time, the coupling strength between nodes increases to 1.224. Therefore, the global adaptive approach is robust to noise. Compared to the results in Fig.1, it is easy to find that the network reaches cluster synchronization after a short time.

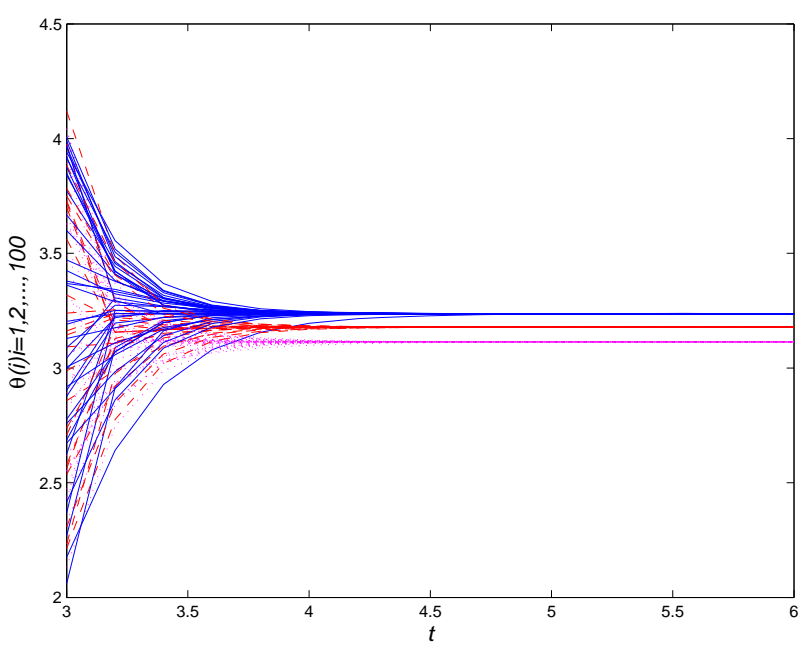

(a)

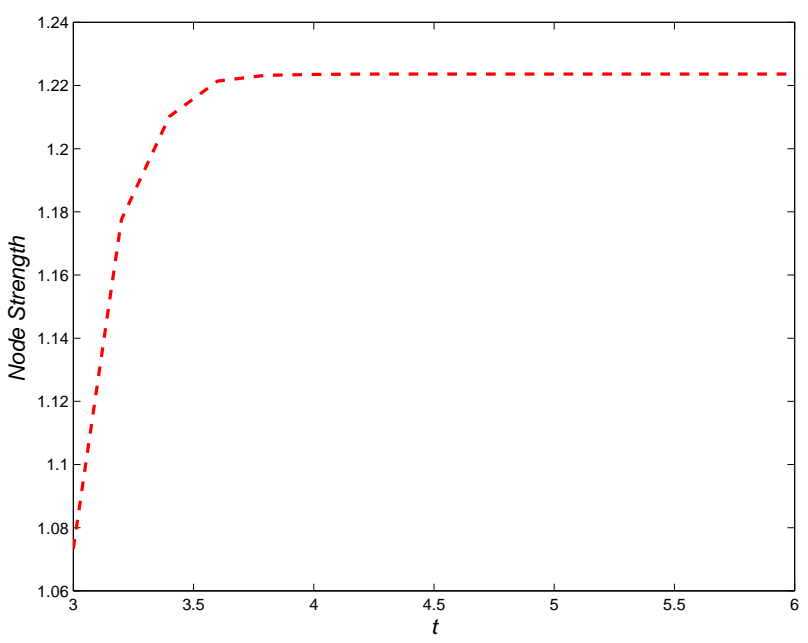

(b)

Fig. 2 Cluster synchronization in the Kuramoto oscillators, where the underlying network is BA scalefree network with noise and the global adaptive gain $\beta=0.1$. (a) $\theta_{i}(t)$ (b)edge strengths $w(t)$. 


\section{$B$ Local adaptive approach in BA scale-free network}

In Fig.3, the same network as that in Fig.1 is used, where the local adaptive approach (6) and the distributed controller (4) are added to each node. As can be seen from Fig. 3(a), for $t>9.5 s, 24$ dashed lines reduce to one dashed line, 38 dotted lines reduce to one dotted line, and the rest 38 solid lines reduce to one solid line. This also implies that the network reaches the desired cluster synchronization. As we know from Fig.1, the network adopted the global adaptive approach reaches cluster synchronization for $t=4.8 \mathrm{~s}$, which is much lower than the 9.5s in Fig.3. Therefore, the same network adopted local adaptive approach has longer transient time than that adopted global adaptive approach.

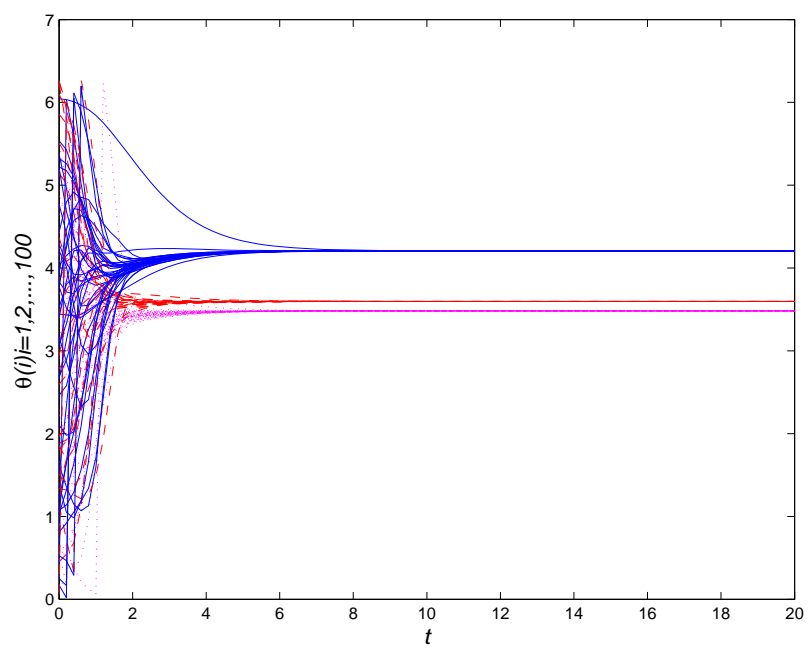

(a)

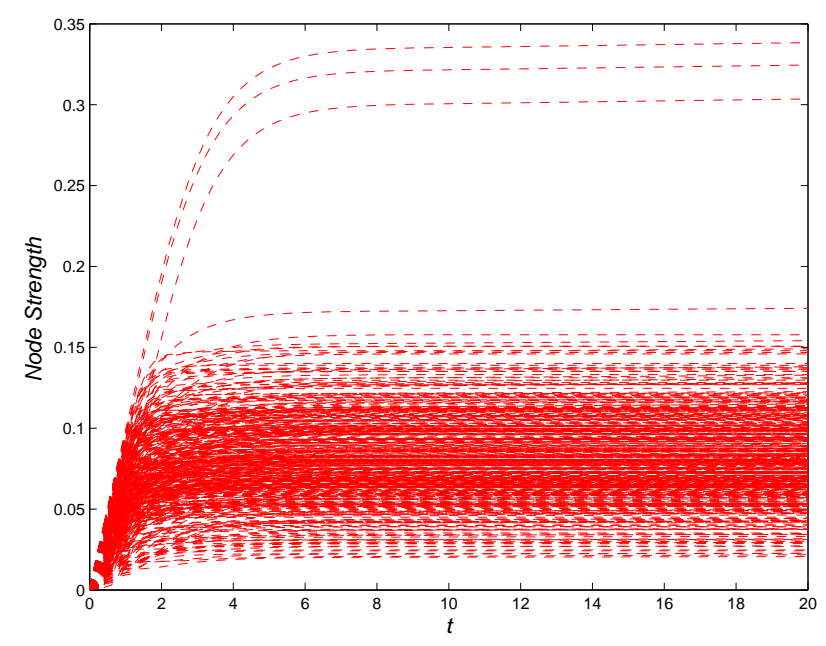

(b)

Fig. 3 Cluster synchronization in the Kuramoto oscillators, where the underlying network is BA scale- free network without noise and the local adaptive gain $\beta=0.1$. (a) $\theta_{i}(t)$ (b)edge strengths $w_{i j}(t)$.

Fig. 3(b) shows the variation of all the edge strength $w_{i j}$ between node $i$ and node $j$ keeps constant for $t>9.5 s$. Combined with Fig. 3(a), it is easy to find that the network reaches cluster synchronization for $t>9.5 \mathrm{~s}$, which implies that the left side of (6) will be zero. Therefore, the edge strength will not change any longer. As shown in Fig.3(a), most edge strengths are lower than 0.15. As shown in Fig.1(b), when the network adopts the global adaptive strength, the same network achieves cluster synchronization for the edge strength equals to 1.073. This means that many unnecessary edge strengths are wasted if the edge strengths do not change with the local adaptive strategy. However, the local adaptive approach must know the local information at each time, which leads that the cost is bigger than that of global adaptive approach.

A same independent noise as that in Fig.2 is added to the variable of (1) respectively at $t=20 \mathrm{~s}$ in Fig. 4 . As shown in Fig.4(a), the network again reaches cluster synchronization for $t=25 \mathrm{~s}$.

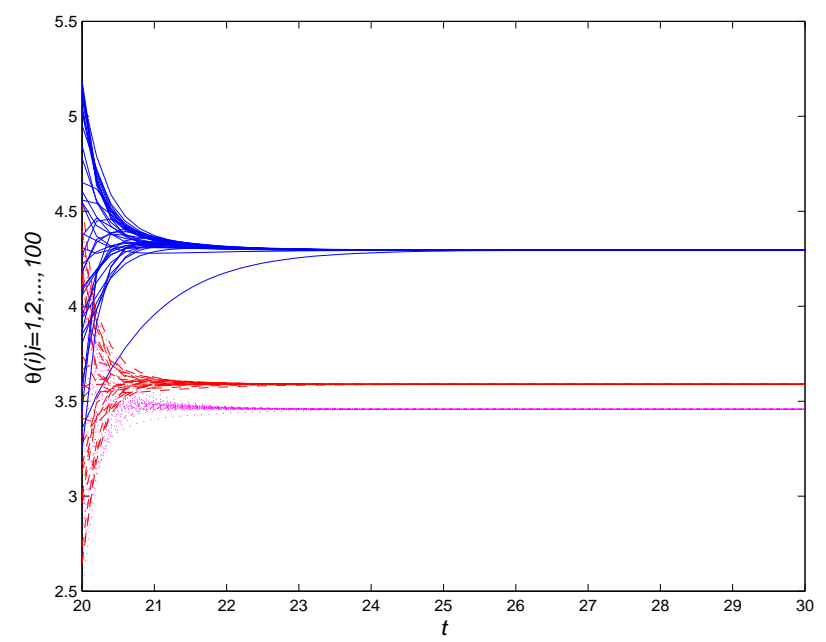

(a) 


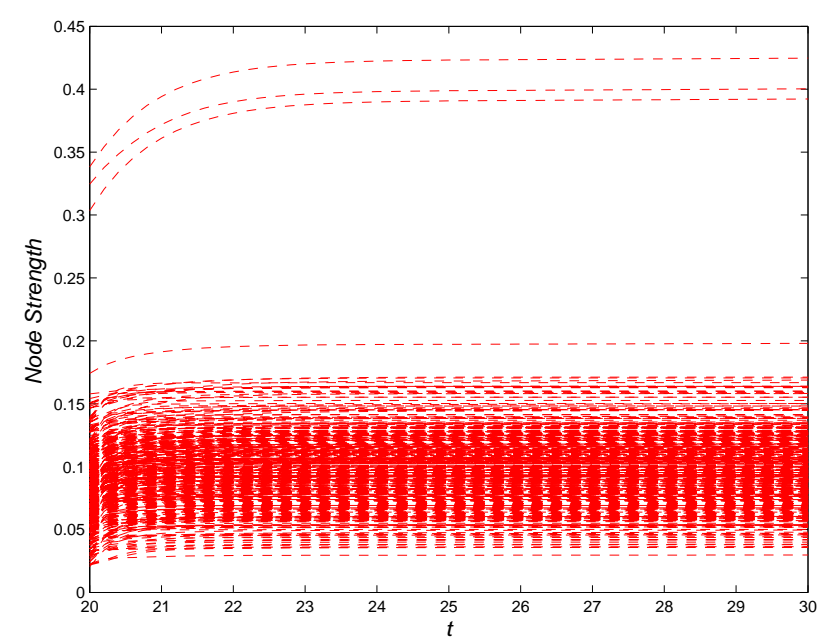

(b)

Fig. 4 Cluster synchronization in the Kuramoto oscillators, where the underlying network is BA scalefree network with noise and the local adaptive gain $\beta=0.1$. (a) $\theta_{i}(t)$ (b)edge strengths $w_{i j}(t)$.

\section{Global adaptive approach in WS small-world network}

A WS small-world network is constructed, where the average degree $2 \mathrm{k}=10$, the rewiring probability is $p=0.2$ and the network size is $N=80$ [17]. The whole nodes of the investigated network are randomly divided into two groups, which means $M=2$. The desired state $s_{i}(t)$ of node $i$ at time $t$ is chosen as the average of the nodes' states in the same group:

$$
\left\{\begin{array}{l}
s_{1}(t)=s_{2}(t)=\cdots=s_{N_{1}}(t)=\frac{\theta_{1}(t)+\cdots+\theta_{N_{1}}(t)}{N_{1}} \\
s_{N_{1}+1}(t)=s_{N_{1}+2}(t)=\cdots=s_{N_{1}+N_{2}}(t)=\frac{\theta_{N_{1}+1}(t)+\cdots+\theta_{N}(t)}{N_{2}}
\end{array}\right.
$$

where two group size $N_{1}$ and $N_{2}$ are 14 and 66, respectively. The nodes' initial phases are randomly distributed in $[0,2 \pi]$ and the initial edge strengths are set to zero.

Fig.5 shows that the cluster synchronization in Kuramoto oscillators, where the underlying network is the typical WS small-world. The distributed controller (4) is added to each node, and the edge strength is adjusted according to the global adaptive appoach (5) with the adaptive gain $\beta=0.1$.
As shown in Fig. 5(a), for $t>3.8 s, 14$ dashed lines reduce to one dashed line, 66 dotted line reduces to one dotted line. This implies that the network reaches the desired cluster synchronization for $t>3.8 \mathrm{~s}$.

It is easy to find that in Fig. 5(b) that the edge strength $w(t)$ keeps constant to be 0.909 for $t>3.8 \mathrm{~s}$. Combined with Fig. 1(a), it is found that the network reaches cluster synchronization for $t>3.8 \mathrm{~s}$, which means that the left side of (5) is zero. Therefore, the edge strength does not increase any more in theory, which is consistent with the simulation result in Fig.5.

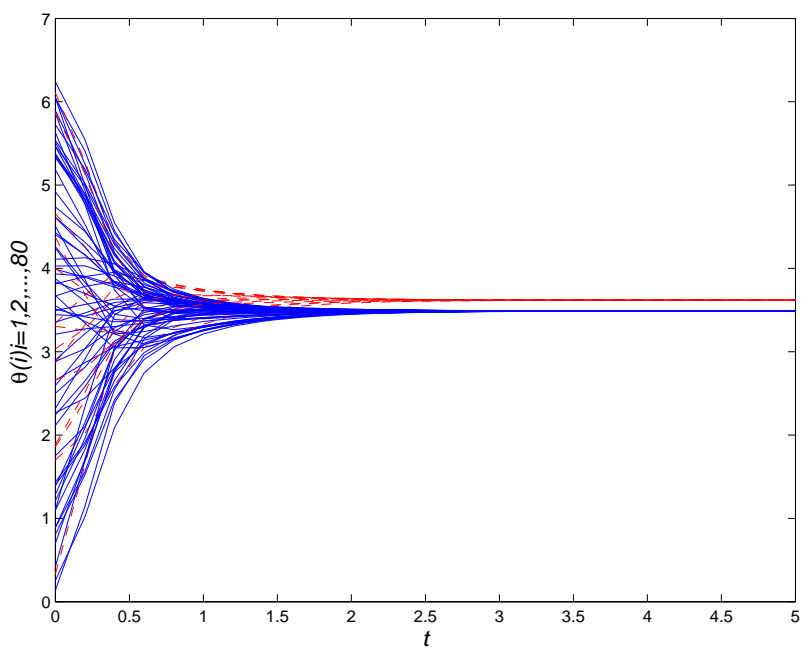

(a)

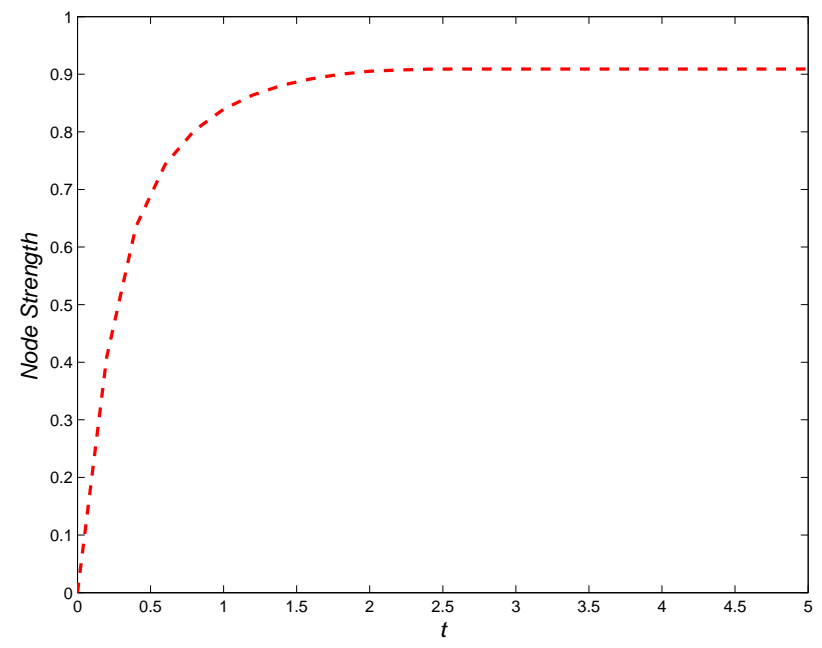

(b)

Fig. 5 Cluster synchronization in the Kuramoto oscillators, where the underlying network is WS smallworld network without noise and the global adaptive gain $\beta=0.1$. (a) $\theta_{i}(t)$ (b)edge strengths $w(t)$. 
An independent noise $\alpha \psi_{i}$ is added to the variable of (1) respectively at $t=3 \mathrm{~s}$ in Fig. 6, where the amplitude of the noise $\alpha$ is $4 ; \psi_{i}$ randomly satisfies the normal Gaussian distribution $N(0,1)$.

As shown in Fig.6(a), the network reaches cluster synchronization again for $t>7 \mathrm{~s}$. At the same time, the coupling strength between nodes increases to 1.187 . Therefore, the global adaptive approach is robust to noise, when the underlying network is WS small-world network.

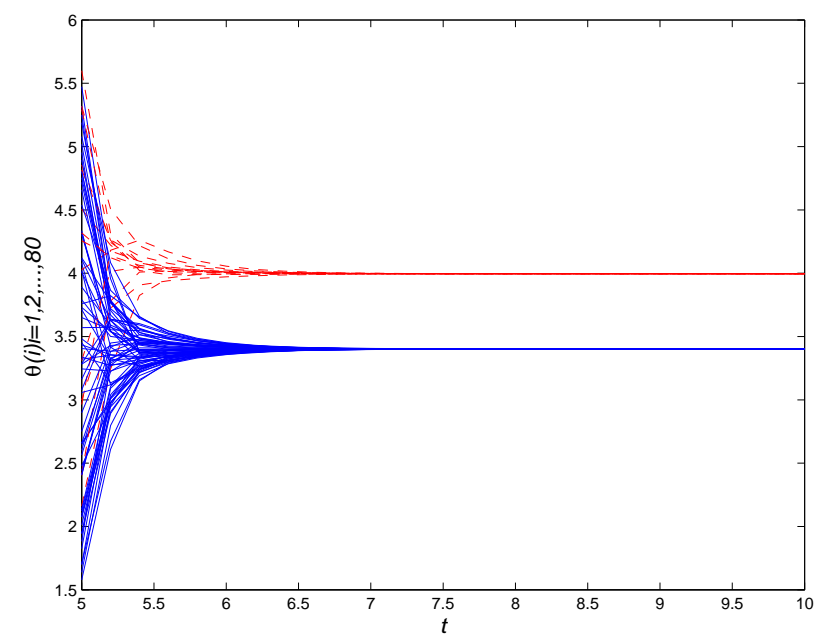

(a)

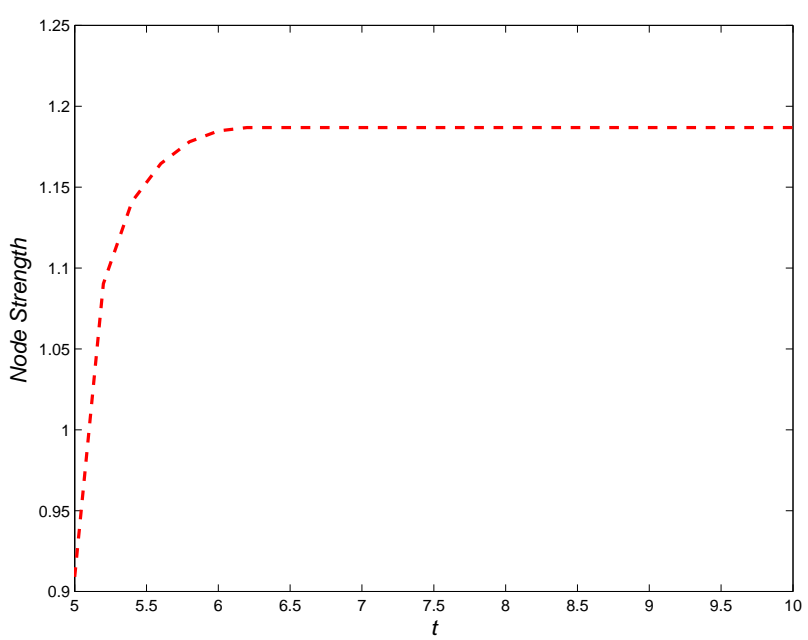

(b)

Fig. 6 Cluster synchronization in the Kuramoto oscillators, where the underlying network is WS smallworld network with noise and the global adaptive gain $\beta=0.1$. (a) $\theta_{i}(t)$ (b)edge strengths $w(t)$.

D Local adaptive approach in WS small-world network
In Fig.7, the same network as that in Fig.5 is used, where the local adaptive approach (6) and the distributed controller (4) are added to each node. As can be seen from Fig. 3(a), for $t>10 \mathrm{~s}, 14$ dashed lines reduce to one dashed line, 66 dotted lines reduce to one dotted line. This also implies that the network reaches the desired cluster synchronization. As we know from Fig.5, the network adopted the global adaptive approach reaches cluster synchronization for $t=7 \mathrm{~s}$, which is much lower than the 10s in Fig.5. Therefore, the same network adopted local adaptive approach has longer transient time than that adopted global adaptive approach.

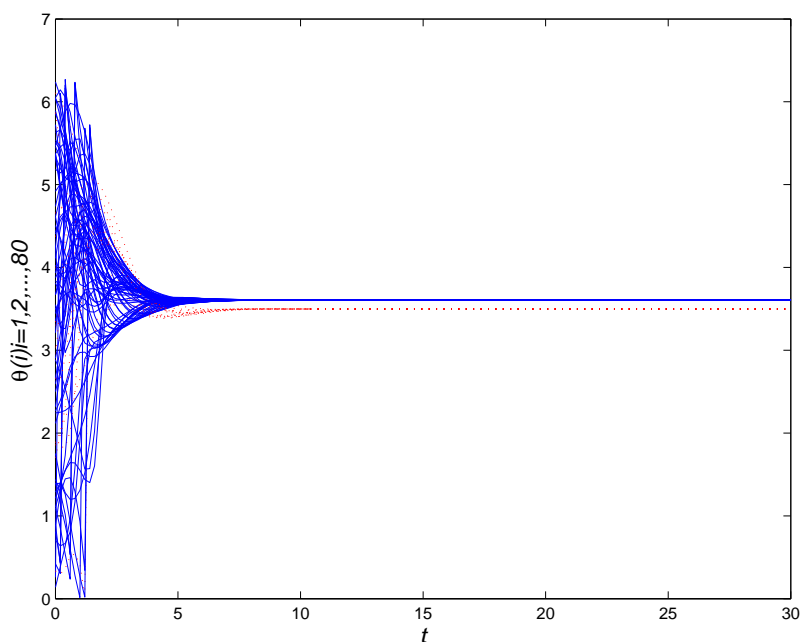

(a)

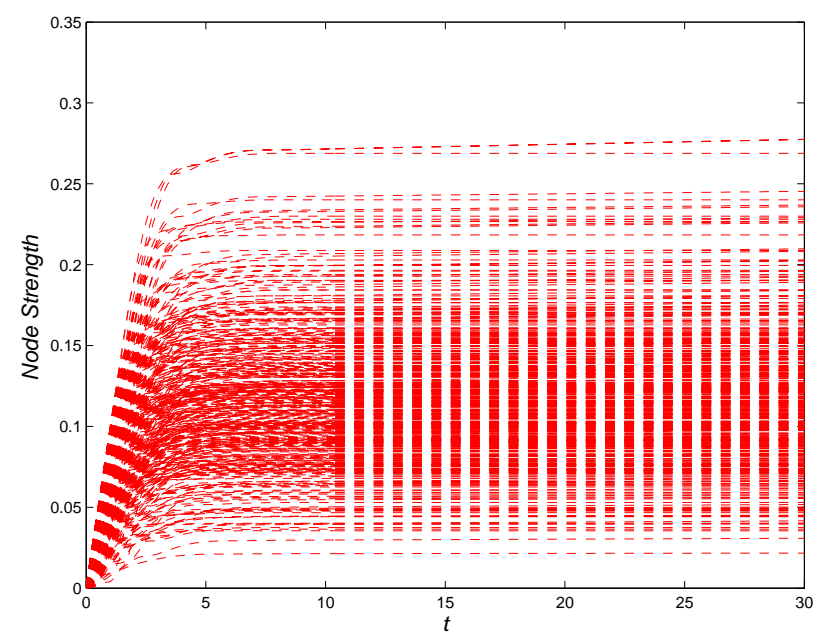

(b)

Fig. 7 Cluster synchronization in the Kuramoto oscillators, where the underlying network is WS smallworld network without noise and the local adaptive gain $\beta=0.1$. (a) $\theta_{i}(t)$ (b)edge strengths $w(t)$. 
Fig. 7(b) shows the variation of all the edge strength $w_{i j}$ between node $i$ and node $j$ keeps constant for $t>10$ s . Combined with Fig. 7(a), it is easy to find that the network reaches cluster synchronization for $t>9.5 \mathrm{~s}$, which implies that the left side of (6) will be zero. Therefore, the edge strength will not change any longer. As shown in Fig.7(a), all the edge strengths are lower than 0.25 , which is much lower than the corresponding strength 0.909 .

A same independent noise as that in Fig.6 is added to the variable of (1) respectively at $t=30 \mathrm{~s}$ in Fig. 8. As shown in Fig.8, the network again reaches cluster synchronization for $t>34 s$.

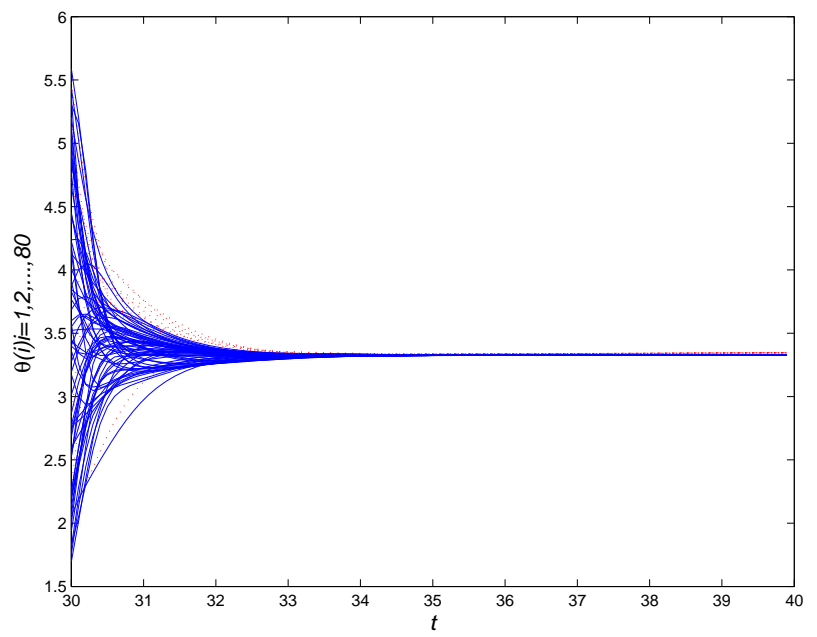

(a)

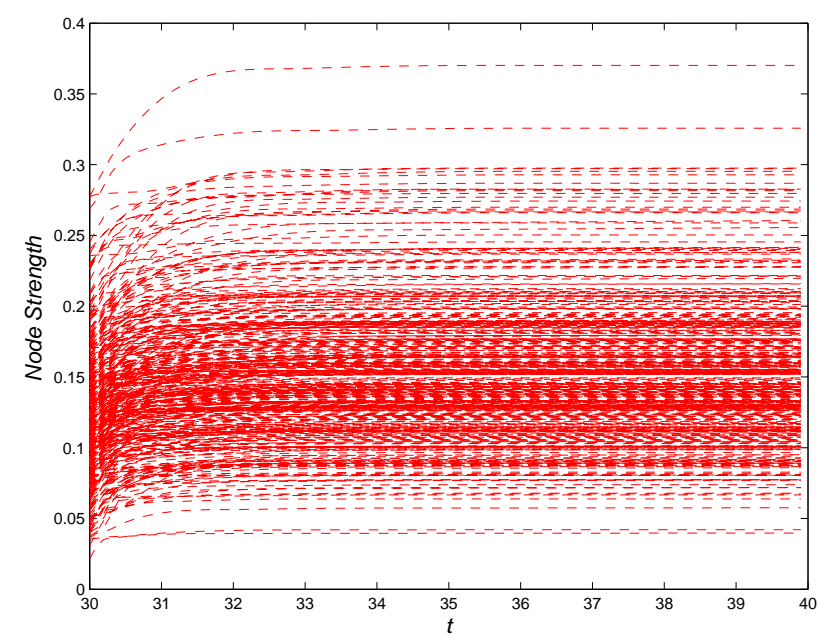

(b)

Fig. 8 Cluster synchronization in the Kuramoto oscillators, where the underlying network is WS small- world network with noise and the local adaptive gain $\beta=0.1$. (a) $\theta_{i}(t)$ (b)edge strengths $w(t)$.

\section{E Adaptive approach in a friendship network}

A friendship network between karate club members is shown in Fig. 9. The network was concluded from the real data collected by Zachary, over a 2-year period of observations [18], which has 34 nodes and 78 edges. It is easy to find that the network has two groups of size 16 and 18, which are represented by circles and squares, respectively.

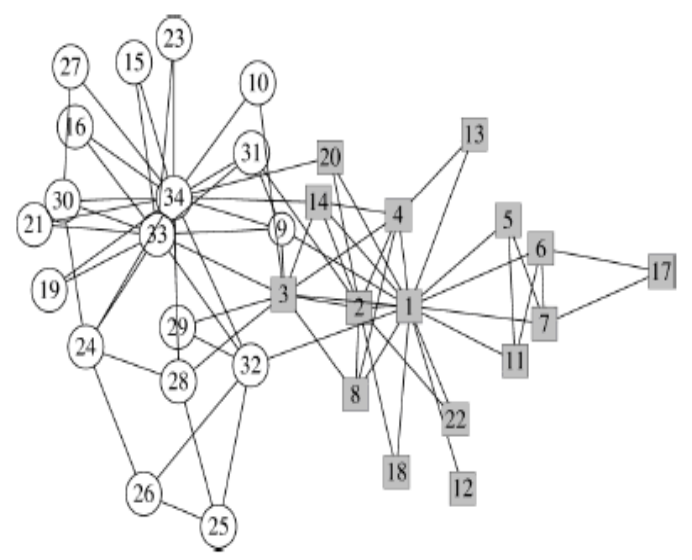

Fig.9 The friendship network between members in karate club [18]

Without loss of generality, the order of the nodes is realigned so that the first group has Node 1 to 16 and the second group has Node 17 to 34 . The desired state $s_{i}(t)$ of node $i$ at time $t$ is chosen as the average of the nodes' states in the same group:

$$
\left\{\begin{array}{l}
s_{1}(t)=s_{2}(t)=\cdots=s_{16}(t)=\left(\theta_{1}(t)+\cdot \cdot+\theta_{16}(t)\right) / 16 \\
s_{17}(t)=s_{18}(t)=\cdots=s_{34}(t)=\left(\theta_{17}(t)+\cdot \cdot+\theta_{34}(t)\right) / 18
\end{array}\right.
$$

where two group size $N_{1}$ and $N_{2}$ are 16 and 18, respectively.

Fig. 10 shows that when the edge strength is adjusted according to the global adaptive approach (5) and the global adaptive gain is 0.1 , the network achieves cluster synchronization for $t>7.8 \mathrm{~s}$. 


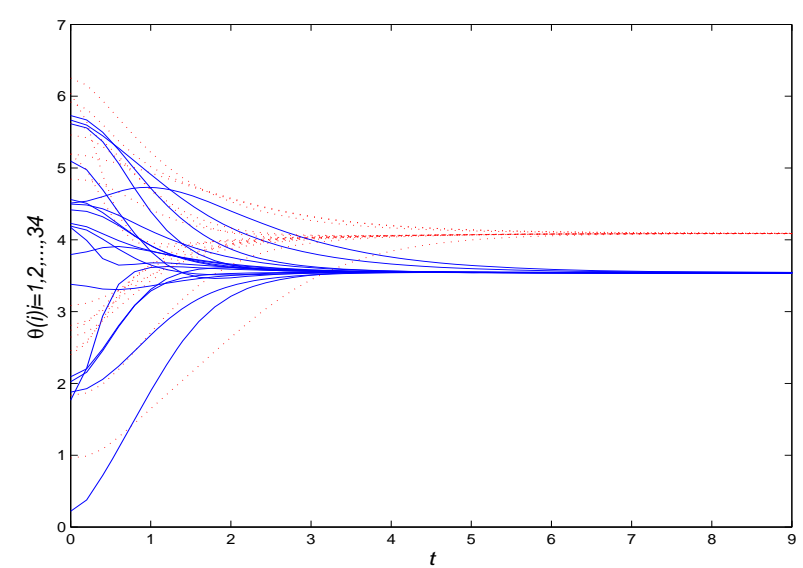

(a)

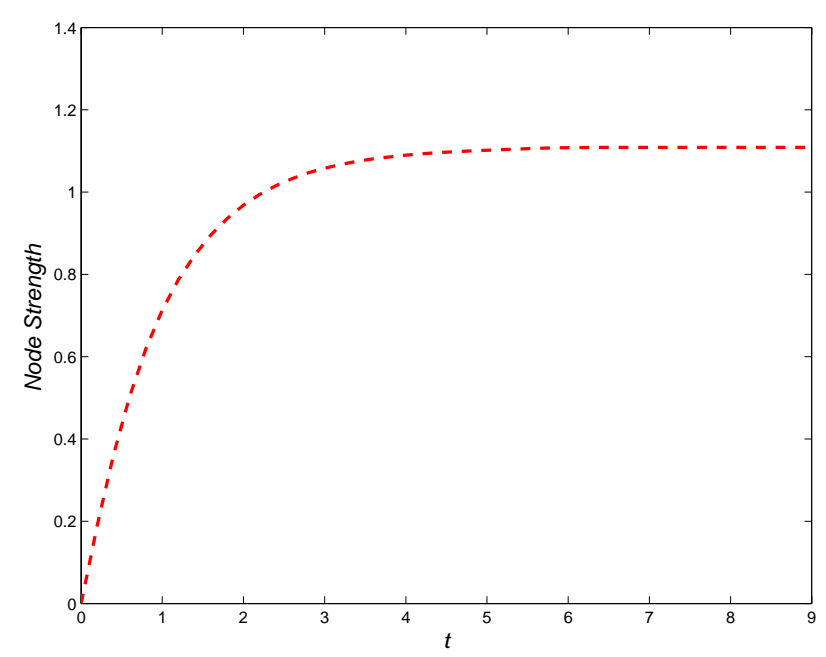

(b)

Fig. 10 Cluster synchronization in the Kuramoto oscillators, where the underlying network is the network shown in Fig.9 and the global adaptive gain $\beta=0.1$. (a)

$\theta_{i}(t)$ (b)edge strengths $w(t)$.

Fig. 11 shows that when the edge strength is adjusted according to the local adaptive approach (6) and the local adaptive gain is 0.1 , the network achieves cluster synchronization for $t>18 \mathrm{~s}$.

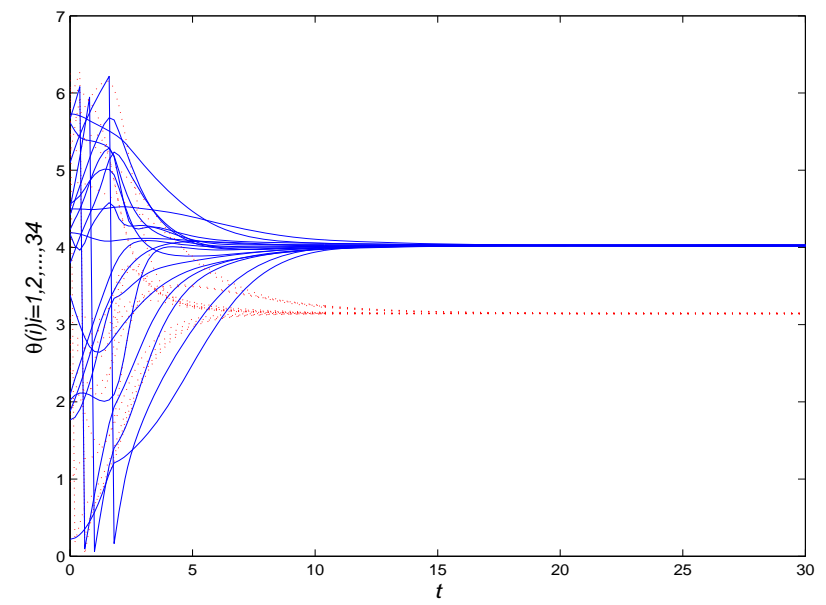

(a)

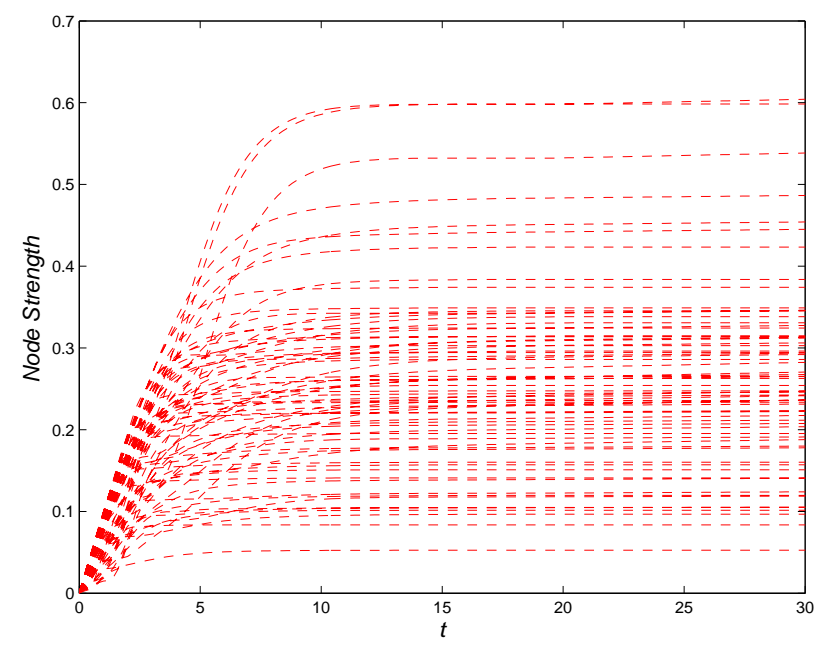

(b)

Fig. 11 Cluster synchronization in the Kuramoto oscillators, where the underlying network is the network shown in Fig.9 and the local adaptive gain $\beta=0.1$. (a)

$\theta_{i}(t)$ (b)edge strengths $w(t)$.

\section{Conclusions}

A novel approach to make a coupled phase oscillators achieve cluster synchronization was proposed. In this approach, the input controller added to each node is based on itself and its neighbors' destination. In addition, the edge strength between connected nodes was adjusted by global adaptive approach and local adaptive approach, respectively. The global adaptive approach used each node's state and its destination; while the local approach uses only local information of related nodes. By two classical network topologies and a real friendship network, the effectiveness was confirmed by numerical simulation. This strategy can be extended to other complex dynamical networks with nonlinear dynamics and will be helpful to comprehend the underlying mechanism of complex dynamical network. 


\section{ACKNOWLEDGMENT}

This work was supported in part by the Fundamental Research Funds for the Central Universities under the Grant 2009B20114.

\section{REFERENCES}

[1] X B Lu, B Z Qin, Synchronization in complex networks, Nova Science Press, New York, 2011.

[2] S. H. Strogatz, "From Kuramoto to Crawford: exploring the onset of synchronization in populations of coupled oscillators”, Physica D, vol.143,pp. 1-20,2000.

[3] K. Kaneko, "Relevance of Clustering to Biological Networks”, Physica D vol. 75, pp.55-73,1994.

[4] N. F Rulkov., "Images of synchronized chaos: Experiments with circuits” Chaos, vol. 6, pp.262-279, 1996.

[5] M. I Rabinovich, et al., "Origin of coherent structures in a discrete chaotic medium” Phys.Rev. E, vol. 60, pp.11301133, 1999.

[6] D. H Zanette, A. S. Mikhailov, "Condensation in globally coupled populations of chaotic dynamical systems", Phys.Rev. E, vol. 57, pp. 276-281, 1998.

[7] W. X Qin., G. Chen,“Coupling schemes for cluster synchronization in coupled Josephson equation”, Physica D, vol.197,pp.375-391,2004.

[8] V. N.Belykh, I.Belykh V., E. Mosekide, "Cluster synchronization modes in an ensemble of coupled chaotic oscillators”, Phys. Rev. E, vol. 63, 036216, 2001.

[9]. S. Jalan, R. E Amrikar., C. K. Hu, "Synchronized clusters in coupled map networks. I. Numerical studies”, Phys. Rev. E, vol. 72, 016211, 2005.

[10] J Kurths., C Zhou., "Dynamical weights and enhanced synchronization in adaptive complex networks", Physical Review Letters, vol.96, 164102, 2006.

[11] T Nishikawa, A E. Motter, "Synchronization is optimal in nondiagonalizable networks”, Physical Review E, vol. 73, 065106, 2006.

[12] J Zhou., J. A Lu., J. Lu, "Pinning adaptive synchronization of a general complex dynamical network", IEEE Transactions on Automatic Control, vol. 51(4), pp. 652-656, 2006.

[13] P. D. Lellis, M. D. Bernardo, F. Garofalo, "Novel decentralized adaptive strategies for the synchronization of complex networks", Automatica, vol.45, pp.1312-1318, 2009.

[14] W L Lu. Chaos, “Adaptive dynamical networks via neighborhood information: Synchronization and pinning control”, vol.17(2), 23122, 2007.

[15] Q. S. Ren, J. Y. Zhao "Adaptive coupling and enhanced synchronization in coupled phase oscillators”, Phys. Rev. E. vol.76 016207, 2007.

[16] A L Barabasi, R Albert. "Emergence of scaling in random networks," Science.vol. 286, pp.509-512, 1999.

[17] D J Watts, S H Strogatz. "Collective dynamics of "smallworld" networks”, Nature. Vol. 393, pp.440-442, 1998.

[18] W. W. Zachary, "An information flow model for conflict and fission in small groups", Journal of anthropological research, vol.33, pp.452-473, 1977.
Xin Biao Lu was born in Shan Dong of China, on November 7, 1975. He graduated from the Shanghai Jiaotong University and works at Hohai University.

His special fields of interest included complex networks analysis and control, cascading failure identify and control of power network.

He received $\mathrm{PhD}$ degree at the major of control theory and control engineering from Shanghai Jiaotong University on June 2008. He received master degree at the major of control theory and control engineering from Shandong University on July 2004. 